\title{
Taxonomy and Systematics/Taxonomia e Sistemåtica Borboletas (Lepidoptera: Papilionoidea e Hesperioidea) da Reserva Biológica do Gurupi, Maranhão, Brasil
}

Registered on ZooBank: urn:lsid:zoobank.org:pub:7398B49E-DAEB-46CD-8FE6-83E1804D2CC4

\author{
Surama Pereira1 ${ }^{1}$, Walisson Mickael Alves Rezende² \& Joseleide Teixeira Câmara ${ }^{3}$
}

1. Programa de Pós Graduação em Biodiversidade e Saúde (PPGBAS), Centro do Estudos Superiores da Universidade Estadual do Maranhão (CESC/ UEMA). 2. Ciências Biológicas Licenciatura, Centro do Estudos Superiores da Universidade Estadual do Maranhão (CESC/UEMA). 3. Universidade Estadual do Maranhão - Centro de Estudos Superiores de Caxias (CESC) - Departamento de Química e Biologia.

\section{EntomoBrasilis 11 (2): 124-138 (2018)}

Resumo. As borboletas constituem um dos grupos mais utilizados em estudos de monitoramento e mensuração do equilíbrio ambiental, uma vez que são sensíveis a qualquer tipo de alteração ambiental. O objetivo do presente estudo é divulgar a primeira lista de espécies de borboletas da REBIO do Gurupi e estimar a representatividade dessas espécies na comunidade. Foram realizadas coletas bimestrais, durante sete dias consecutivos, entre jan/2010 e jul/2011. Os espécimes foram obtidos com uso de rede entomológica e armadilhas do tipo Van Someren-Rydon. Na análise dos dados foram calculados a frequência das espécies, índices de diversidade, estimativas de riqueza e obteve-se a curva de acúmulo de espécies. Foram obtidos 413 espécimes de 91 espécies, 64 gêneros, 14 subfamílias e seis famílias. A espécie mais frequente foi Morpho menelaus (Linnaeus, 1758). Os índices de diversidades obtidos, quando comparados a outros estudos, são expressivos; a estimativa de espécies e a curva de acúmulo mostram que a comunidade amostrada está subestimada. Com esse trabalho, o Maranhão alcançou 358 espécies de borboletas registradas. Os dados ressaltam a importância da REBIO e das demais Unidades de Conservação para a manutenção da riqueza de borboletas da região.

Palavras-chave: Amazônia Maranhense; Borboleta; Conservação; Diversidade; Monitoramento Ambiental.

\section{Butterflies (Lepidoptera: Papilionoidea and Hesperioidea) from the Gurupi Biological Reserve, Maranhão State, Brazil}

\begin{abstract}
Butterflies are one of the groups most used in monitoring and measuring environmental balance studies, as they are sensitive to any type of environmental change. The objective of the present study is to disclose the first list of butterfly species of REBIO Gurupi and to estimate the representativeness of these species in the community. Were performed collected quarterly during seven consecutive days, between Jan/2010 and Jul/ 2011. The specimens were obtained using an entomological network and traps of the Van Someren-Rydon type. In the analysis of the data were calculated the frequency of species, diversity indexes, wealth estimates and the curve of species accumulation was obtained. The most frequent species was Morpho menelaus (Linnaeus, 1758). The diversity indexes obtained, when compared to other studies, are expressive; The species estimates and the accumulation curve show that the sampled community is underestimated. With this work, Maranhão reached 358 species of registered butterflies. The data emphasizes the importance of REBIO and other conservation units for the maintenance of the butterflies' wealth of the region.
\end{abstract}

Keywords: Amazonia Maranhense; Butterfly; Conservation; Diversity; Wildlife inventory.

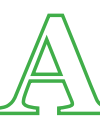

tualmente a diversidade biológica vem sendo destruída em ritmo acelerado e a causa principal da redução das populações é a destruição de seu habitat natural. A crescente ameaça às poucas áreas naturais que ainda restam, a necessidade de identificação de grupos indicadores para o monitoramento ambiental como os insetos têm sido cada vez mais urgentes (BROWN JR \& FrEITAS 2000).

Dentre os domínios brasileiros, a Amazônia e a Mata Atlântica são destaques por serem florestas tropicais que possuem altíssima diversidade biológica e ao mesmo tempo, estão entre os ecossistemas mais ameaçados do mundo. Significativas ações antrópicas vêm sendo impostas a estes: implantação de grandes áreas urbanas, exploração madeireira, desmatamento, queimadas, fragmentação e a mineração, além da invasão de espécies exóticas, tráfico de animais silvestres e as mudanças climáticas (BROWN-JR 1996).

Os insetos são indispensáveis para uso em estudos de avaliação de impacto ambiental e de efeitos de fragmentação florestal, pois além de ser o grupo de animais mais diverso do planeta, com elevadas densidades populacionais, apresentam grande diversidade, em termos de espécies e de habitats, grande variedade de habilidades para dispersão e seleção de hospedeiros, de respostas à qualidade e quantidade de recursos disponíveis, além de sua dinâmica populacional ser altamente influenciada pela heterogeneidade dentro de um mesmo habitat. Também são importantes pelo seu papel no funcionamento dos

\section{Edited by:}

William Costa Rodrigues

\section{Article History:}

Received: o6.i.2018

Accepted: 19.iv.2018
Corresponding author:

Joseleide Teixeira Câmara

○ jtcamara75@gmail.com

(3) http://orcid.org/0000-0002-0736-1306
Funding agencies:

\&NPq/MCTI-Amazônia Oriental 
ecossistemas naturais atuando como predadores, parasitos, fitófagos, saprófagos, polinizadores, entre outros (SouzA \& BROWN 1994).

Dentre os insetos, as borboletas constituem um dos grupos mais utilizados em estudos de monitoramento e mensuração do equilíbrio ambiental (BROWN $J_{R}$ 1997). Esses insetos reúnem um conjunto de características que permitem seu uso para tal finalidade: são abundantes e diversos na maioria dos ecossistemas, são facilmente coletados com armadilhas iscadas com frutos fermentados, possuem taxonomia relativamente bem conhecida e são considerados excelentes bioindicadores, respondendo rapidamente as alterações que ocorrem nos ecossistemas naturais.

As borboletas se mostram melhores indicadoras para fragmentação da paisagem (UEHARA-PRADO et al. 2007). Também estão no "ranking" de espécies guarda-chuva ou espécies bandeira, ou seja, a preservação das populações de borboletas de um determinado ecossistema implica na preservação de outros grupos de organismos desse mesmo ecossistema.

A Reserva Biológica (REBIO) do Gurupi constitui o maior fragmento remanescente de Floresta Amazônica no Maranhão, é a única unidade de conservação dessa natureza nesse Estado e faz parte de uma região que atualmente vem sendo bastante degradada pela ação antrópica, principalmente por desmatamentos para o cultivo de plantações, além da retirada de madeira ilegal (Oliveira 2011). Dentro das ações conservacionistas, estudos de diversidade e levantamento faunístico têm sido considerados de caráter prioritário para essa UC. Uma vez que estes são fundamentais para a avaliação e o monitoramento da perda em biodiversidade, principalmente das borboletas que são organismos frágeis, bem como para o desenvolvimento de diferentes tipos de pesquisas e estratégias de conservação desses insetos e de seu habitat.

Este estudo é de fundamental importância para avaliar a diversidade e possibilitar futuros estudos de monitoramento para a região. O objetivo principal do presente estudo é divulgar a primeira lista de espécies de borboletas da REBIO do Gurupi e estimar a representatividade dessas espécies na comunidade.

\section{MATERIAL E MÉTODOS}

A Reserva Biológica (REBIO) do Gurupi está localizada na Amazônia Oriental, ao noroeste do Estado do Maranhão, entre as bacias do Rio Gurupi e Pindaré, é a única unidade de conservação (UC) dessa categoria no Estado (SNUC 2005). A mesma apresenta grande importância para a conservação ambiental dessa floresta, localiza-se no Centro de Endemismo Belém e integra a lista de áreas prioritárias para a conservação (Almeida \& Vieira 2010).

A REBIO foi criada pelo decreto $\mathrm{n}^{0} 95.614$ de 12 de janeiro de 1988, possui uma área com aproximadamente de 271 mil ha, abrangendo os municípios de Bom Jardim, Centro Novo do Maranhão e São João do Caru (Moura et al. 2011) (Figura 1).

Foram realizadas coletas bimestrais, durante sete dias consecutivos, entre jan/2010 e jul/2011, para as campanhas foram utilizados dois pontos alternados: um ponto ao Norte ( $\left.3^{\circ} 11^{\prime} 37^{\prime \prime} \mathrm{S} ; 46^{\circ} 44^{\prime} 39^{\prime \prime} \mathrm{W}\right)$ e outro ponto ao Sul ( $4^{\circ} \mathrm{O} 5^{\prime} 22^{\prime \prime} \mathrm{S}$; $\left.46^{\circ} 50^{\prime} 30^{\prime \prime} \mathrm{W}\right)$ da Reserva. Os espécimes foram obtidos com uso de rede entomológica, em trilhas e estradas entorno dos acampamentos e, com armadilhas do tipo Van Someren-Rydon, iscadas com uma mistura de frutas fermentadas. O processo de identificação, o posicionamento sistemático adotado e a distribuição das espécies dentro dos gêneros e tribos seguem LAMAS (2004). Todo material foi preparado a seco, montado em alfinetes entomológicos e encontra-se depositado na Coleção Zoológica do Maranhão (CZMA), localizada na Universidade Estadual do Maranhão (UEMA), campus Caxias.

As espécies da REBIO do Gurupi compõem a lista de borboletas registradas para o Estado do Maranhão, que é apresentada em anexo em conjunto com os dados de Bates (1867), Garcia et al.

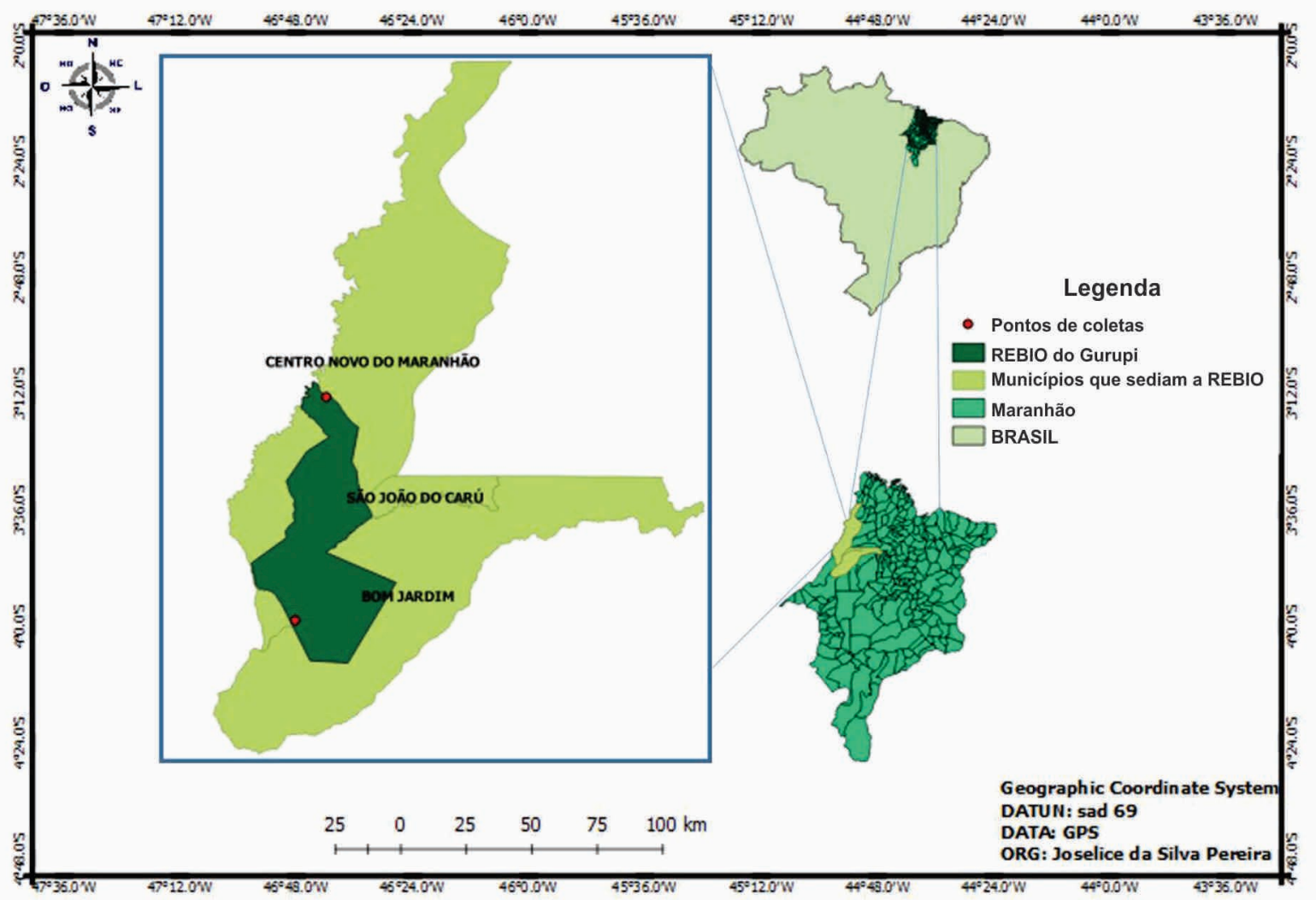

Figura 1. Mapa da REBIO do Gurupi, com pontos de coletas. 
(1990), Garcia \& Bergmann (1994), Ramos (2000) e Martins et al. (2017).

Na análise dos dados, calculou-se a frequência das espécies segundo Silveira-Neto et al. (1976), foram obtidos os índices de diversidade de Simpson (S') e de Shannon-Wiener (H'), as estimativas de riqueza de espécies empregando os procedimentos "Bootstrap", "Chao1", "Chao2", "Jackknife1", "Jackknife2" com utilização dos pacotes BiodiversityR e vegan do software de livre acesso, sistema estatístico R (R Core TeAm 2016). Também foi construída a curva de acúmulo de espécies utilizando o software citado anteriormente.

\section{RESULTADOS E DISCUSSÃO}

Foram obtidos 413 espécimes de 91 espécies, distribuídos dentro de 64 gêneros, 14 subfamílias e seis famílias (Tabela 1).

Tabela 1. Listas de espécies de borboletas (Hesperioidea e Papilionoidea) registradas para REBIO do Gurupi, no Maranhão, no período de janeiro de 2010 a julho de 2011. $\mathrm{N}=$ número de espécimes, FR.= Frequência relativa, R. E. = Rede Entomológica, Arm. Armadilha Van Someren-Rydon.

\begin{tabular}{l}
\hline \multicolumn{1}{|c|}{ Família /Subfamília/Espécies } \\
\hline Nymphalidae \\
Biblidinae \\
\hline Catonephele acontius (Linnaeus, 1771) \\
Catonephele numilia (Cramer 1775) \\
Diaethria clymena (Cramer, 1775) \\
Hamadryas feronia Linnaeus, 1758 \\
Marpesia orsilochus (Fabricius, 1776) \\
Nessaea batesii (C. Felder \& R. Felder, 1860) \\
Nessaea obrinus (Linnaeus, 1758) \\
Nica flavilla Godart, 1824 \\
Temenis laothoe Cramer, 1777 \\
Brassolinae
\end{tabular}
N.
FR.

Método de coleta R.E.

Arm.

Bia actorion actorion (Linnaeus, 1763)

Caligo idomeneus (Linnaeus, 1758)

Caligo illioneus (Cramer 1775)

Opsiphanes invirae (Hübner, [1808])

Opsiphanis quiteria (Stoll, 1780)

Charaxinae

Archaeoprepona demophoon (Hübner, [1814])

Fountainea halice chrysophana (H. Bates, 1866)

Fountainea ryphea (Cramer, 1775)

Hipna clytemnestra Cramer, 1777

Memphis glauce glauce (Felder \& Felder, 1862)

1
1
5
3
1
4
2
10

\section{0,2}

0,2

0,5

1,2

0,7

0,2

1,0

0,5

2,4

Memphis leonida (Stoll, 1782)

Memphis oenomais (Boisduval, 1870)

Prepona laertes (Hübner, [1811])

Mesoprepona pheridamas (Cramer, 1777)

Zaretis isidora (Cramer, 1779)

\section{Heliconiinae}

Eueides lybia (Fabricius, 1775)

Heliconius erato (Linnaeus, 1758)

Heliconius numata (Cramer, 1780)

Heliconius sara (Fabricius, 1793)

Heliconius sp.

Heliconius wallacei flavescens Weymer, 1891

Philaethria dido dido (Linnaeus, 1763)

\section{Ithomiinae}

Ceratinia sp.

Episcada sp.

Hypothyris euclea (Godart, 1819)

Hypothyris sp.

Mechanitis lysimnia (Fabricius, 1793)

Mechanitis polymnia (Linnaeus, 1758)

Methona sp.

Napeogenes sp.

Oleria sp.

Scada sp.

\section{Morphinae}

Morpho helenor Cramer, 1776

Morpho menelaus (Linnaeus, 1758)

Morpho deidamia Hübner, 1819

Nymphalinae

Colobura dirce (Linnaeus, 1758)

Historis acheronta (Fabricius, 1775)
1

2

2

2

1

1

11

4

6

6

14

7

0,0

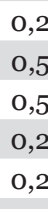

2,7

1,0

1,5

3,4

1,7

2,4

0,2

1,0

0,5

0,5

2

6

10

1

6

1

3

1

1

12

19

8

1

1

1

4
1

7

7
1

\section{1}

41

1

2

4

0,2

2,9

4,6

1,9

0,2

0,2

1,0

0,2

1,7

0,2

2,7

9,9

0,2

0,5

1,0

\begin{tabular}{ll}
1,5 & $\mathrm{x}$ \\
2,4 & $\mathrm{x}$ \\
0,2 & $\mathrm{x}$ \\
1,5 & $\mathrm{x}$ \\
0,2 & $\mathrm{x}$ \\
0,7 & $\mathrm{x}$ \\
0,2 & $\mathrm{x}$ \\
\hline
\end{tabular}

$\mathrm{x}$
$\mathrm{x}$
$\mathrm{x}$
$\mathrm{x}$
$\mathrm{X}$
$\mathrm{X}$
$\mathrm{x}$
$\mathrm{x}$
$\mathrm{x}$
$\mathrm{X}$

$\mathrm{x}$

$\mathrm{x}$

X

X

$\mathrm{x}$

$\mathrm{X}$

$\mathrm{X}$

X

$\mathrm{x}$

X

X

$\mathrm{x}$

$\mathrm{x}$

$\mathrm{X}$

$\mathrm{X}$

X

$\mathrm{x}$

$\mathrm{X}$

X

$\mathrm{X}$

$\begin{array}{ll} & \mathrm{x} \\ \mathrm{x} & \mathrm{x} \\ \mathrm{x} & \mathrm{x} \\ \mathrm{x} & \\ \mathrm{x} & \text { Continua... }\end{array}$


Tabela 1. Continuação...

\begin{tabular}{|c|c|c|c|c|}
\hline \multirow{2}{*}{ Família /Subfamília/Espécies } & \multirow{2}{*}{$\mathbf{N}$. } & \multirow{2}{*}{ FR. } & \multicolumn{2}{|c|}{ Método de coleta } \\
\hline & & & R.E. & Arm. \\
\hline Historis odius (Fabricus, 1775) & 2 & 0,5 & & $\mathrm{x}$ \\
\hline Junonia evarete (Cramer, 1779) & 2 & 0,5 & $\mathrm{x}$ & $\mathrm{x}$ \\
\hline \multicolumn{5}{|l|}{ Satyrinae } \\
\hline Caeruleuptychia coelestis Butler, 1867 & 1 & 0,2 & & $\mathrm{x}$ \\
\hline Chloreuptychia herseis (Godart, [1824]) & 2 & 0,5 & & $\mathrm{x}$ \\
\hline Cissia myncea (Cramer, 1780) & 7 & 1,7 & & $\mathrm{x}$ \\
\hline Cissia penelope (Fabricius, 1775) & 9 & 2,2 & & $\mathrm{x}$ \\
\hline Hermeuptychia hermes (Fabricius, 1775) & 6 & 1,5 & & $\mathrm{x}$ \\
\hline Magneuptychia alcinoe (C. \& R. Felder, 1867) & 1 & 0,2 & & $\mathrm{x}$ \\
\hline Pareuptychia ocirrhoe (Fabricius, 1776) & 34 & 8,2 & $\mathrm{x}$ & $\mathrm{x}$ \\
\hline Paryphthimoides undulata (Butler, 1867) & 5 & 1,2 & $\mathrm{x}$ & \\
\hline Taygetis cleopatra Felder \& Felder, 1867 & 4 & 1,0 & & $\mathrm{x}$ \\
\hline Taygetis echo (Cramer, 1775) & 11 & 2,7 & & $\mathrm{x}$ \\
\hline Taygetis thamyra (Cramer, 1779) & 11 & 2,7 & $\mathrm{x}$ & \\
\hline Taygetis virgilia (Cramer, 1776) & 16 & 3,9 & $\mathrm{x}$ & $\mathrm{x}$ \\
\hline \multicolumn{5}{|l|}{ Hesperiidae } \\
\hline \multicolumn{5}{|l|}{ Pyrginae } \\
\hline Aguna asander (Hewitson, 1867) & 2 & 0,5 & $\mathrm{x}$ & \\
\hline Aguna sp. & 1 & 0,2 & $\mathrm{x}$ & \\
\hline Augiades crinisus (Cramer, 1780) & 2 & 0,5 & $\mathrm{x}$ & \\
\hline Chrysoplectrum perniciosus (Herrich-Schäffer, 1869) & 1 & 0,2 & $\mathrm{x}$ & \\
\hline Nascus phocus (Cramer, 1777) & 1 & 0,2 & $\mathrm{x}$ & \\
\hline Nascus sp. & 2 & 0,5 & $\mathrm{x}$ & \\
\hline Phocides sp. & 1 & 0,2 & $\mathrm{x}$ & \\
\hline Urbanus proteus (Linnaeus, 1758) & 1 & 0,2 & $\mathrm{x}$ & \\
\hline Urbanus simplicius (Stoll, 1790) & 1 & 0,2 & $\mathrm{x}$ & \\
\hline \multicolumn{5}{|l|}{ Lycaenidae } \\
\hline \multicolumn{5}{|l|}{ Polymmatinae } \\
\hline Arawacus sp. & 17 & 4,1 & $\mathrm{x}$ & \\
\hline Evenus sp. & 1 & 0,2 & $\mathrm{x}$ & \\
\hline \multicolumn{5}{|l|}{ Papilionidae } \\
\hline \multicolumn{5}{|l|}{ Papilioninae } \\
\hline Heraclides thoas (Linnaeus, 1771) & 2 & 0,5 & $\mathrm{x}$ & \\
\hline Mimoides sp. & 1 & 0,2 & $\mathrm{x}$ & \\
\hline Parides sp. & 3 & 0,7 & $\mathrm{x}$ & \\
\hline Protesilaus sp. & 1 & 0,2 & $\mathrm{x}$ & \\
\hline \multicolumn{5}{|l|}{ Pieridae } \\
\hline \multicolumn{5}{|l|}{ Coliadinae } \\
\hline Anteos menippe (Hübner, [1818]) & 2 & 0,5 & $\mathrm{x}$ & \\
\hline Aphrissa statira (Cramer, 1777) & 2 & 0,5 & $\mathrm{x}$ & \\
\hline Eurema agave (Cramer, 1775) & 1 & 0,2 & $\mathrm{x}$ & \\
\hline Eurema albula (Cramer, 1775) & 5 & 1,2 & $\mathrm{x}$ & \\
\hline Eurema elathea (Cramer, 1777) & 1 & 0,2 & $\mathrm{x}$ & \\
\hline Phoebis argante (Fabricius, 1775) & 1 & 0,2 & $\mathrm{x}$ & \\
\hline \multicolumn{5}{|l|}{ Pierinae } \\
\hline Glutophrissa drusilla (Cramer, 1777) & 2 & 0,5 & $\mathrm{x}$ & \\
\hline Ascia monuste (Linnaeus, 1764) & 1 & 0,2 & $\mathrm{x}$ & \\
\hline \multicolumn{5}{|l|}{ Riodinidae } \\
\hline \multicolumn{5}{|l|}{ Riodininae } \\
\hline Amarynthis meneria (Cramer, 1776 & 1 & 0,2 & $\mathrm{x}$ & \\
\hline Apodemia sp. & 2 & 0,5 & $\mathrm{x}$ & \\
\hline Calydna sp. & 1 & 0,2 & $\mathrm{x}$ & \\
\hline Charis sp. & 1 & 0,2 & $\mathrm{x}$ & \\
\hline Setabis sp. & 1 & 0,2 & $\mathrm{x}$ & \\
\hline Stalachtis sp. & 3 & 0,7 & $\mathrm{x}$ & \\
\hline Theope pieridoides C. Felder \& R. Felder, 1865 & 1 & 0,2 & $\mathrm{x}$ & \\
\hline Theope sp. & 1 & 0,2 & $\mathrm{x}$ & \\
\hline Total & 413 & 100 & & \\
\hline
\end{tabular}

A família Nymphalidae foi representada com maior riqueza e abundância, com 60 espécies (66\%) e 350 espécimes (85\%). A expressiva riqueza encontrada para Nymphalidae deve-se ao fato desta família possuir o maior número de espécies entre os lepidópteros diurnos e constitui o grupo mais diverso entre as borboletas da Região Neotropical, além disso, possui uma ampla distribuição geográfica. As comunidades de ninfalídeos neotropicais vivem em ambientes formados por um mosaico de paisagens constituído por diferentes tipos de sistemas antrópicos, interligados com habitats primitivos de vários tamanhos, formas e estado de perturbação e, estão distribuídas de forma não uniforme (LAMAS 2004). 
Dentre os ninfalídeos, Satyrinae foi mais representativa em termos de riqueza e abundância (12 espécies e 107 indivíduos), seguida por Charaxinae (10 sp. e 61 ind.). Modelo de representatividade semelhante foi verificado em um fragmento de floresta ombrófila mista, no Rio Grande do Sul (PEDRotTi et al. 2011); na comunidade de borboletas do Horto Botânico Irmão Teodoro Luís, também do Rio Grande do Sul (Silva et al. 2013). Na maioria dos estudos que relacionam borboletas frugívoras, Satyrinae constitui o grupo mais rico e abundante, por exemplo, são assim as comunidades do Parque Metropolitano de Pituaçu, Salvador-BA (VAsconcelos et al. 2009); em uma Área de Proteção Especial (APE) Manancial Cercadinho no sul do município de Belo Horizonte (Silva et al. 2012); e em uma Área de Proteção Especial Manancial Mutuca Nova Lima, Minas Gerais (Silva et al. 2015). Satyrinae possui uma ampla diversidade, biologia e distribuição, compõe um terço de todas as espécies de Nymphalidae, suas principais plantas hospedeiras são monocotiledôneas (PEÑa \& WAHLBERg 2008). No entanto, as diferentes metodologias e esforços amostrais utilizadas nestes estudos dificultam comparações diretas.

Morpho menelaus (Linnaeus, 1758) obteve maior abundância entre as espécies (9,9\%). Essa espécie é sensível ao desmatamento e fragmentação florestal, os adultos necessitam de significativos espaços florestados (MARTins et al. 2014). Ou seja, a alta frequência dessa espécie pode estar associada àboa disponibilidade de recurso alimentar que favorece o estabelecimento e desenvolvimento dos espécimes na área de estudo, além disso, segundo Brown \& FREITAS (2000) é uma espécie considerada indicadora de áreas importantes para a conservação e está relacionada a fatores ambientais como topografia e vegetação complexas.

Os índices de diversidade de Shannon-Wiener (H') e Simpson (S') encontrados para a amostra total foram 3,90 e 0,97, respectivamente. CAMARGO (1999), em estudo sobre a composição e a diversidade de lepidópteros noturnos em cinco áreas de Cerrado (nos estados do Maranhão, Bahia e Minas Gerais), listou 1.064 espécies de 33 famílias e obteve $\mathrm{H}^{\prime}=3,48$ e $\mathrm{S}^{\prime}=0,79$. $\mathrm{Na}$ comunidade de borboletas da Floresta Estacional Decidual, no município de Santa Maria no estado do Rio Grande do Sul, foram coletadas 89 espécies e o H'=3,6 (SAckis \& Morais 2008). Para a comunidade de borboletas na Reserva Biológica do Lami, no município de Viamão, Rio Grande do Sul, área bastante ameaçada pela ocupação humana, obteve-se H'=3,06 (MARCHIORI et al. 2007).

As estimativas de riqueza para a amostra de borboletas da REBIO do Gurupi variaram de 111 espécies (Bootstrap) a 183 (Chao 2) (Tabela 2). Portanto, o número de espécies listadas nesse estudo (91 espécies) representa entre 50\% e 82\% da real riqueza estimada para a área de estudo.

Tabela 2. Estimadores de riqueza obtidos para borboletas (Hesperioidea e Papilionoidea) da REBIO do Gurupi, no Maranhão, obtidas no período de janeiro de 2010 a julho de 2011.

\begin{tabular}{lc}
\hline Variáveis & N/médias \\
\hline Amostras & 9 \\
Espécies & 91 \\
\hline ACE & 140 \\
Bootstrap & 111 \\
Chao1 & 126 \\
Chao2 & 183 \\
\hline Jackknife1 & 139 \\
\hline Jackknife2 & 171 \\
\hline
\end{tabular}

As estimativas obtidas corroboram com a curva de acumulação de espécies construída (Figura 2), que não apresenta uma tendência assíntota, indicando que possivelmente com a continuidade de coletas, muitas espécies devem ser adicionadas ao número amostrado. Para Giovenardi $e t$ al. (2008), os resultados também indicam insuficiência amostral para alcançar um nível estável. No entanto, a estabilização destas curvas em ambientes tropicais

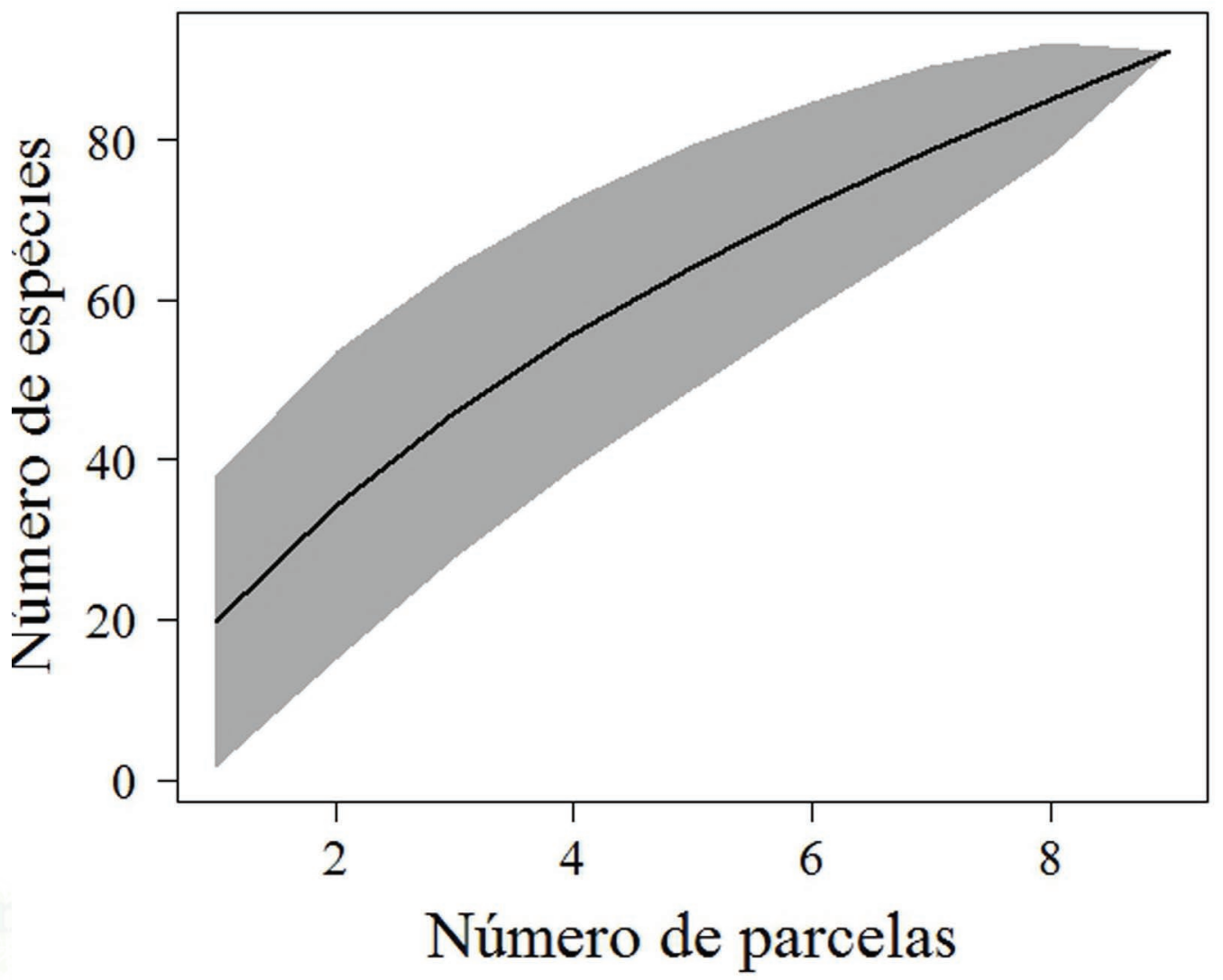

Figura 2. Curva de acúmulo de espécie de borboletas (Lepidoptera: Hesperioidea e Papilionoidea) da REBIO do Gurupi, capturadas no período de janeiro de 2010 a julho de 2011. 
com altíssima diversidade, trata-se de um fato particularmente difícil (SANTOS 2003).

Outro fato que constitui uma problemática na mensuração da real riqueza da área de estudo é a continuidade de coletas sistemáticas na área de estudo. A equipe que realizou as campanhas, só entrava na área com apoio e escolta da Polícia Militar, dadas as circunstâncias de perigo por conta das disputas violentas entre madeireiros, grileiros e outros exploradores dos recursos naturais da UC.

Com base nos dados obtidos em estudos anteriores realizados no Estado do Maranhão (BATES 1867; GARCIA et al. 1990; GARCIA \& Bergmann 1994; Ramos 2000; Martins et al. 2017), o presente estudo acrescenta 50 novos registros, totalizando 358 espécies de borboletas para o Estado do Maranhão, incluindo borboletas frugívoras e nectarívoras (Tabela 3 - Anexo). Vale ressaltar que o Maranhão constitui um território amplo e possui um mosaico de ecossistemas, fato que sugere o potencial de altíssima riqueza de borboletas para esta região.

A composição da comunidade de borboletas da REBIO do Gurupi possui características que sugerem uma área com recursos naturais em boas condições para manutenção das populações de borboletas. No entanto, sabe-se que a intensa ação antrópica na área de estudo poderá alterar estas condições rapidamente.

Apesar das análises mostrarem que a lista de espécies apresentada está longe de representar a real riqueza total de borboletas da REBIO do Gurupi, o presente estudo constituivaliosa contribuição científica do ponto de vista da conservação da biodiversidade dessa área, pois se trata do primeiro registro do grupo para esta UC, possibilitando assim o monitoramento da comunidade e mensuração de impactos ambientais nessa comunidade.

O crescente aumento da fronteira agrícola no Estado do Maranhão, além dos históricos problemas de desmatamento da região amazônica e mais recentemente do Cerrado (outro domínio importante no território maranhense), são problemas que ameaçam diariamente a biodiversidade da área. Por tanto, esforços devem ser empreendidos para a continuidade e ampliação dos pontos de coletas em todo o território maranhense, especialmente em áreas de transição entre a Amazônia e o Cerrado, locais que possuem alta heterogeneidade e fragilidade de ambiente.

\section{AGRADECIMENTOS}

Os autores agradecem ao Dr. Olaf Hermann Hendrik Mielke e à Dra. Mirna Martins Casagrande (UFPR), pela sua contribuição na identificação dos espécimes; ao Programa de Pesquisa em Biodiversidade (PPBio) e CNPq/MCTI-Amazônia Oriental (Processo 558287/2009-3) pelo financiamento da pesquisa e coleta; ao ICMBio, pela autorização da pesquisa na REBIO Gurupi; e aos colegas Jocifran Alves Silva, Rosilda Rodrigues Carvalho e Dr. Francisco Limeira de Oliveira, por suas colaborações durante as coletas.

\section{REFERÊNCIAS}

Almeida, A.S. \& I.C.G. Vieira, 2010. Centro de Endemismo de Belém: status da vegetação remanescente e desafios para a conservação da biodiversidade e restauração ecológica. Revista de Estudos Universitários, 36: 95-111.

Bates, H.W., 1867. On a collection of butterflies formed by Thomas Belt, Esq., in the interior of the province of Maranhão, Brazil. Transactions of the Royal Entomological Society of London, $3^{\circ}$ série (5- parte vii): $335-546$

Brown Jr., K.S. \& A.V.L. Freitas, 2000. Diversidade de Lepidoptera em Santa Teresa, Espírito Santo. Boletim do Museu de Biologia Mello Leitão, 11/12: 71-118.
Brown Jr., K.S., 1997. Diversity, disturbance and sustainable use of neotropical forests: insects as indicators for conservation monitoring. Journal of Insect Conservation, 1: 25-42.

Brown-Jr., K.S., 1996. Conservation of threatened species of Brazilian butterflies, p 45-62. In: Ae, S.A., T. Hirowatari, M. Ishii \& L.P. Brower (Eds.). Decline and conservation of butterflies in Japan. Yadoriga special issue. Lepidopterist Society of Japan, Osaka. 317 p.

Camargo, A.J.A., 1999. Estudo comparativo sobre a composição e a diversidade de lepidópteros noturnos em cinco áreas da Região dos Cerrados. Revista Brasileira de Zoologia, 16: 369-380. DOI: https://doi.org/10.1590/s010181751999000200004.

Garcia, I.P. \& Bergmann, E.C., 1994. Borboletas da ilha de São Luis (MA). Arquivos do Instituto Biológico, 56: 37-38.

Garcia, I.P., Bergmann, E.C. \& S.M. Rodrigues-Neto, 1990. Diversidade mensal de borboletas na ilha de São Luis (MA). Arquivos do Instituto Biológico, 57: 39-44.

Giovenardi, R., R.A.D. Mare, J. Sponchiado, S.H. Roani, F.A.F. Jacomassa, A.B. Jung \& M.A. Porn, 2008. Diversidade de Lepidoptera (Papilionoidea e Hesperioidea) em dois fragmentos de floresta no município de Frederico Westphalen, Rio Grande do Sul, Brasil. Revista Brasileira de Entomologia, 52: 599-605. DOI: https://doi.org/10.1590/ s0085-56262008000400010.

Lamas, G., 2004. Hesperioidea-Papilionoidea, p. 1-439. In: Heppner, J. (Ed.). Atlas of Neotropical Lepidoptera. Association for Tropical Lepidoptera, Scientinfic Publishers, $479 \mathrm{p}$.

Marchiori, M.O., M.O. Teixeira, E.C. Teixeira \& H.P. Romanowski, 2007. Riqueza, composição e diversidade de borboletas (Lepidoptera, Papilionoidea e Hesperioidea) em ambientes de restinga no entorno do lago Guaíba, RS, Brasil. In: VIIICEB -Sociedade de Ecologia do Brasil, Caxumbu, Minas Gerais. p. $1-3$.

Martins, D. S., P.S.F. Ferreira, M.J. Fornazier \& J. S. Santos, 2014. Insetos da Reserva Natural Vale, Linhares, Espírito Santo, Brasil. Ciência \& Ambiente, 49: 219-235.

Martins, L.P., E.C. Araujo Junior, A.R.P. Martins, M.S. Colins, G.C.F. Almeida \& G.G. Azevedo, 2017. Butterflies of Amazon and Cerrado remnants of Maranhão, Northeast Brazil. Campinas/SP. Biota Neotropica, 1: 1-12. DOI: https://doi.org/10.1590/1676-0611-bn-2017-0335.

Moura, W.C., J.C., Fukuda, E.A., Lisboa, B.N. Gomes, S.L. Oliveira, M.P. Santos, A.S. Carvalho \& M.B. Martins, 2011. A Reserva Biológica do Gurupi como instrumento de conservação da Amazônia Oriental, p. 25-34. In: Martins, M B. \& T. G. de. Oliveira (Eds.), Amazônia Maranhense: Diversidade e Conservação. Belém: MPEG, 328 p.

Oliveira, T.G., 2011. Estado de Conservação da Reserva Biológica do Gurupi: alerta vermelho à conservação da última fronteira amazônica do Maranhão, São Luís, MA, p. 282-295. In: Martins M.B. \& T.G. Oliveira (Eds.). Amazônia Maranhense: diversidade e conservação. Belém: Museu Paraense Emílio Goeldi, 328 p.

Pedrotti, V.S., M.P. Barros, H.P. Romanowski \& C.A. Iserhard, 2011. Borboletas frugívoras (Lepidoptera, Nymphalidae) ocorrentes em um fragmento de Floresta Ombrófila Mista no Rio Grande do Sul, Brasil. Biota Neotropica, 11: 385-390. DOI: https://doi.org/10.1590/s1676-06032011000100036.

Peña, C. \& N. Wahlberg, 2008. Prehistorical climate change increased diversification of a group of butterflies. Biology Letters, 4: 274-278. DOI: https://doi.org/10.1098/ rsbl.2008.0062.

Pereira, H.A., J.R.B., Silva, G.D.V. Marques, E. Germanos, A.J.A. Camargo \& V. Stefani, 2011. Levantamento de lepidópteros (Insecta: Lepidoptera) diurnos em Mata Mesófila Semi Decídua de um Fragmento Urban. Bioscience Journal, 27: 839-848. 
R Core Team, 2016. R: A language and environment for statistical computing. R Foundation for Statistical Computing, Vienna, Austria. Disponível em: <https://www.r-project.org/>.

Ramos, F.A., 2000. Nymphalidae butterfly communities in an Amazonian forest fragment. Journal of Research on the Lepidoptera, 35: 29-41.

Sackis, G.D. \& A.B.B. Morais, 2008. Borboletas (Lepidoptera: Hesperioidea e Papilionoidea) do campus da Universidade Federal de Santa Maria, Santa Maria, Rio Grande do Sul. Biota Neotropica, 8: 151-158. DOI: https://doi.org/10.1590/ s1676-06032008000100018.

Santos, A.J. 2003. Estimativas de riqueza de espécies, p. 19-41. In: Cullen Jr., L., R. Rudran \& C. Valladares-padua (Org). Métodos de estudos em Biologia da Conservação \& Manejo da Vida Silvestre, Curitiba, Editora da UFPR, 667 p.

Silva, A.R.M., C.O. Castro, P.O. Mafia, M.O.C. Mendonça, T.C.C. Alves \& M.V. Beirão, 2012. Borboletas frugívoras (Lepidoptera: Nymphalidae) de uma área urbana (Área de Proteção Especial Manancial Cercadinho) em Belo Horizonte, Minas Gerais. Biota Neotropica, 12: 293-297. DOI: https://doi.org/10.1590/s1676-06032012000300028.

Silva, A.R.M., D.V. Pontes, M.P. Guimaraes, M.V. Oliveira, L.T.F. Assis \& M. Uehara-Prado, 2015. Fruit-feeding butterflies (Lepidoptera: Nymphalidae) of the Area de Proteção Especial Manancial Mutuca, Nova Lima and Species list for the Region of Belo Horizonte, Minas Gerais, Brazil. Biota Neotropica, 15: e20140118. DOI: https://doi.org/10.1590/167606032015011814.

ANEXO

Tabela 3. Lista de espécies de borboletas (Hesperioidea e Papilionoidea) registradas para o Maranhão.

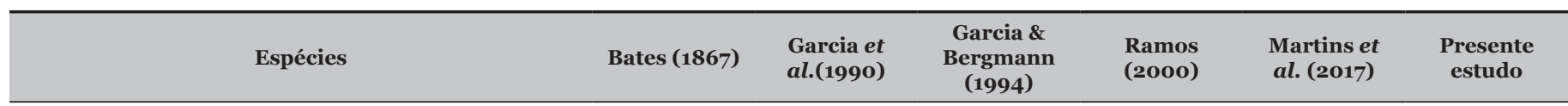

\section{HESPERIIDAE}

\section{Eudaminae}

Aguna asander asander (Hewitson, 1867)

Aguna metophis (Latreille, [1824])

Aguna sp.

Astraptes fulgerator fulgerator (Walch, 1775)

Autochton neis (Geyer, 1832)

Chioides catillus catillus (Cramer, 1779)

Epargyreus clavicornis clavicornis (Herrich-Schäffer, 1869)

Euriphellus euribates (Stoll, 1782)

Hyalothyrus leucomelas (Geyer, 1832)

Phocides pigmalion hewitsonius (Mabille, 1883)

Typhedanus crameri McHenry, 1960

Udranomia orcinus (C. Felder \& R. Felder, 1867)

Urbanus chalco (Hübner, 1823)

Urbanus dorantes dorantes (Stoll, 1790)

Urbanus procne (Plötz, 1881)

Urbanus proteus (Linnaeus, 1758)

Urbanus simplicius (Stoll, 1790)

Urbanus sp. 1 $\mathrm{x}$

Urbanus sp. 2

Hesperiinae

Aides aegita (Hewitson, 1866)

Aides duma argyrina Cowan, 1970

Aides duma duma Evans, 1955

Augiades crinisus (Cramer, 1780)

Calpodes ethlius (Stoll, 1782)
Silva, J.M, S.K. Cunha, E.J.E Silva \& F.R.M. Garcia, 2013. Borboletas frugívoras (Lepidoptera: Nynphalidae) no Horto Botanico Irmão Deodoro Luís, Capão do Leão, Rio Grande do Sul. Revista Biotemas, 26: 87-95. DOI: https://doi.org/10.5007/2175-7925.2013v26n1p87.

Silveira-Neto, S., O. Nakano, D. Barbin \& N.A. Vila Nova, 1976. Manual de ecologia dos insetos. São Paulo, Agronômica Ceres, $420 \mathrm{p}$.

SNUC, 2005. Sistema Nacional de Unidades de Conservação. Lei No 9.985, de 18 de julho de 2000. Disponível em: $<$ http://www.mma.gov.br/port/sbf/dap/doc/snuc.pdf $>$.

Souza, O.F.F. \& V.K. Brown, 1994. Effects of habitat fragmentation on Amazonian termite communities. Journal of Tropical Ecology, 10: 197-206. DOI: https://doi.org/10.1017/ s0266467400007847.

Uehara-Prado, M., K.S. Brown Jr \& A.V.L. Freitas, 2007. Species richness, composition and abundance of fruit-feeding butterflies in the Brazilian Atlantic Forest: comparison between a fragmented and continuous landscape. Global Ecology and Biogeography, 16: 43-54. DOI: https://doi.org/10.1111/j.1466-822x.2006.00267.x.

Vasconcelos, R.N., E.C.C. Barbosa \& M.C.L. Peres. 2009. Borboletas do parque metropolitano de Pituaçu, Salvador, Bahia, Brasil. Sitientibus, 9: 158-164. 
Tabela 3. Continuação...

\begin{tabular}{|c|c|c|c|c|c|c|}
\hline Espécies & Bates (1867) & $\begin{array}{l}\text { Garcia et } \\
\text { al.(1990) }\end{array}$ & $\begin{array}{c}\text { Garcia \& } \\
\text { Bergmann } \\
(1994)\end{array}$ & $\begin{array}{l}\text { Ramos } \\
(2000)\end{array}$ & $\begin{array}{l}\text { Martins et } \\
\text { al. (2017) }\end{array}$ & $\begin{array}{c}\text { Presente } \\
\text { estudo }\end{array}$ \\
\hline Carystoides basoches (Latreille, [1824]) & & & & & $\mathrm{x}$ & \\
\hline Carystoides maroma (Möschler, 1877) & & & & & $\mathrm{x}$ & \\
\hline Carystus phorcus phorcus (Cramer, 1777) & & & & & $\mathrm{x}$ & \\
\hline Cobalus calvina (Hewitson, 1866) & & & & & $\mathrm{x}$ & \\
\hline Cymaenes tripunctus theogenis (Capronnier, 1874) & & & & & $\mathrm{x}$ & \\
\hline Cynea irma (Möschler, 1879) & & & & & $\mathrm{x}$ & \\
\hline Cynea robba robba Evans, 1955 & & & & & $\mathrm{x}$ & \\
\hline Enosis uza uza (Hewitson, 1877) & & & & & $\mathrm{x}$ & \\
\hline Hesperia heliopetes Hewitson & & $\mathrm{x}$ & $\mathrm{x}$ & & & \\
\hline Hesperia orcas Cramer & & $\mathrm{x}$ & $\mathrm{x}$ & & & \\
\hline Mnasicles hicetaon Godman, 1901 & & & & & $\mathrm{x}$ & \\
\hline Morys valerius (Möschler, 1879) & & & & & $\mathrm{x}$ & \\
\hline Nyctelius nyctelius nyctelius (Latreille, [1824]) & & & & & $\mathrm{x}$ & \\
\hline Panoquina fusina fusina (Hewitson, 1868) & & & & & $\mathrm{x}$ & \\
\hline Panoquina ocola ocola (Edwards, 1863) & & & & & $\mathrm{x}$ & \\
\hline Phanes aletes (Geyer, 1832) & & & & & $\mathrm{x}$ & \\
\hline Polites vibex (Geyer, 1832) & & & & & $\mathrm{x}$ & \\
\hline Pompeius pompeius (Latreille, [1824]) & & & & & $\mathrm{x}$ & \\
\hline Pyrrhopyge sp. & & $\mathrm{x}$ & & & & \\
\hline Pyrrhopygopsis socrates (Ménétriés, 1855) & & & & & $\mathrm{x}$ & \\
\hline Saliana saladin Evans, 1955 & & & & & $\mathrm{x}$ & \\
\hline Synale hylaspes (Stoll, 1781) & & & & & $\mathrm{x}$ & \\
\hline
\end{tabular}

\section{Pyrginae}

Augiades crinisus (Cramer, 1780)

Chrysoplectrum perniciosus (Herrich-Schäffer, 1869)

Elbella sp.

Grais stigmaticus stigmaticus (Mabille, 1883)

Heliopetes arsalte (Linnaeus, 1758)

Heliopetes omrina (Butler, 1870)

Jemadia fallax fida Evans, 1951

Mysoria barcastus antila Evans, 1951

Nascus phocus (Cramer, 1777)

Nascus sp.

Olina stalachtoides

Phocides sp.

Pyrgus orcus (Stoll, 1780)

Pyrgus veturius Plötz, 1884

Pyrrhopyge phidias (Linnaeus, 1758)

Pyrrhopyge sp. Hübner, [1819]

Timochares trifasciata (Hewitson, 1868)

Timochreon satyrus (C. Felder \& R. Felder, 1867)

Urbanus proteus (Linnaeus, 1758)

Urbanus simplicius (Stoll, 1790)

Viola violella (Mabille, 1898)

\section{NYMPHALIDAE}

\section{Biblidinae}


Tabela 3. Continuação...

\begin{tabular}{|c|c|c|c|c|c|c|}
\hline Espécies & Bates (1867) & $\begin{array}{l}\text { Garcia et } \\
\text { al.(1990) }\end{array}$ & $\begin{array}{c}\text { Garcia \& } \\
\text { Bergmann } \\
(1994)\end{array}$ & $\begin{array}{l}\text { Ramos } \\
\text { (200o) }\end{array}$ & $\begin{array}{l}\text { Martins et } \\
\text { al. (2017) }\end{array}$ & $\begin{array}{c}\text { Presente } \\
\text { estudo }\end{array}$ \\
\hline Callicore maimuna (Hewison, 1858) & & & & $\mathrm{x}$ & & \\
\hline Callicore pygas thamiras (Ménétriés, 1857) & & & & $\mathrm{x}$ & & \\
\hline Catagramma pyracmon peristera Hewitson, 1853 & & & & $\mathrm{x}$ & & \\
\hline Catagramma sp. & & $\mathrm{x}$ & & & & \\
\hline Catonephele acontius (Linnaeus 1771) & & & & $\mathrm{x}$ & $\mathrm{x}$ & $\mathrm{x}$ \\
\hline Catonephele numia (Cramer, 1775) & & & & $\mathrm{x}$ & & $\mathrm{x}$ \\
\hline Diaethria clymena (Cramer, 1755) & & & & $\mathrm{x}$ & & $\mathrm{x}$ \\
\hline Ectima iona E. Doubleday, [1848] & & & & & $\mathrm{x}$ & \\
\hline Ectima thecla lirina Felder \& Felder, 1867 & & & & $\mathrm{x}$ & & \\
\hline Eunica maja (Fabricius, 1775) & & & & & $\mathrm{x}$ & \\
\hline Eunica tatila (Herrich-Schäfer, 1885) & & & & $\mathrm{x}$ & & \\
\hline Hamadryas amphinome (Linnaeus, 1767) & & $\mathrm{x}$ & & $\mathrm{x}$ & $\mathrm{x}$ & \\
\hline Hamadryas chloe (Stoll, 1787) & & & & $\mathrm{x}$ & $\mathrm{x}$ & \\
\hline Hamadryas februa (Hübner, [1823]) & & $\mathrm{x}$ & & $\mathrm{x}$ & $\mathrm{x}$ & \\
\hline Hamadryas februa ferentina(Godart, [1824]) & & & $\mathrm{x}$ & & & \\
\hline Hamadryas feronia (Linnaeus, 1758) & & $\mathrm{x}$ & & $\mathrm{x}$ & $\mathrm{x}$ & $\mathrm{x}$ \\
\hline Hamadryas feronia farinulenta (Fruhstorfer, 1916) & & & $\mathrm{x}$ & $\mathrm{x}$ & & \\
\hline Hamadryas iphthime (Bates, 1864) & & & & $\mathrm{x}$ & & \\
\hline Hamadryas laodamia (Cramer, 1777) & & & & $\mathrm{x}$ & $\mathrm{x}$ & \\
\hline Hamadryas velutina (Bates, 1865) & & & & $\mathrm{x}$ & & \\
\hline Marpesia orsilochus (Fabricius, 1776) & & & & & $\mathrm{x}$ & \\
\hline Myscelia hypatia Strecker, 1900 & & & & $\mathrm{x}$ & & \\
\hline Nessaea batesii (C. Felder \& R. Felder, 186o) & & & & & & $\mathrm{x}$ \\
\hline Nessaea obrinus (Linnaeus, 1758) & & & & $\mathrm{x}$ & & $\mathrm{x}$ \\
\hline Nica flavilla (Godart, [Godart, 1824]) & & & & & & $\mathrm{x}$ \\
\hline Pyrrhogyra neaerea (Linnaeus, 1758) & & & & $\mathrm{x}$ & & \\
\hline Pyrrhogyra otolais H. Bates, 1864 & & & & $\mathrm{x}$ & & \\
\hline Temenis laothoe (Cramer, 1777) & & & & $\mathrm{x}$ & $\mathrm{x}$ & $\mathrm{x}$ \\
\hline Vila azeca stalachtoides (H. Bates, 1867) & & & & $\mathrm{x}$ & & \\
\hline
\end{tabular}

Amphidecta pignerator (A. Butler, 1867)

Bia actorian (Linnaeus, 1767)

Brassolis sophorae (Linnaeus, 1758)

Caligo illioneus (Cramer, 1775)

Caligo teucer (Linnaeus, 1758)

Caligopsis seleucida (Hewitson, 1877)

Catoblepia berecynthia (Cramer, 1777)

Dynamine aff. arene

Dynamine agacles (Dalman, 1823)

Dynamine arene (Hübner, 1823)

Dynamine myrson myrson (Doubleday, 1849)

Dynamine paulina (Bates, 1865)

Dynamine postverta postverta (Cramer, 1779)

Dynamine sp. 1

$\mathrm{x}$

Dynamine sp. 2

Eryphanis polixena (Meerb, 1775)

Narope cyllabarus Westwood, 1851

Narope panniculus Stichel, 1904

Opsiphanes invirae (Hübner, [1808])

Opsiphanes quiteria Stoll, 1780

$\begin{array}{lll}x & x \\ x & x & x \\ x & x & x \\ x & x & x \\ x & x & x \\ & x & x \\ & x & x \\ & x & x \\ & x & x \\ & x & x \\ & x & x\end{array}$

$\mathrm{x}$ 
Tabela 3. Continuação...

\begin{tabular}{|c|c|c|c|c|c|c|}
\hline Espécies & Bates (1867) & $\begin{array}{l}\text { Garcia et } \\
\text { al.(1990) }\end{array}$ & $\begin{array}{c}\text { Garcia \& } \\
\text { Bergmann } \\
\text { (1994) }\end{array}$ & $\begin{array}{l}\text { Ramos } \\
(2000)\end{array}$ & $\begin{array}{c}\text { Martins et } \\
\text { al. (2017) }\end{array}$ & $\begin{array}{c}\text { Presente } \\
\text { estudo }\end{array}$ \\
\hline
\end{tabular}

Opsiphanes sp. 1

$\mathrm{x}$

Opsiphanes sp. 2

\section{Charaxinae}

Anaea sp.

$\mathrm{x}$

Archaeoprepona demophon (Linnaeus, 1758)

Archaeoprepona demophoon (Hübner, [1814])

Archaeoprepona meander (Cramer, 1775)

Fountainea halice chrysophana (H. Bates, 1866)

Fountainea ryphea (Cramer, 1775)

Hypna clytemnestra (Cramer, 1777)

Memphis acidalia (Hübner, [1819])

Memphis aenomais (Boisduval, 1870)

Memphis arachne arachne Cramer, 1777

Memphis glauce glauce (Felder \& Felder, 1862)

Memphis leonida (Stoll, 1782)

Memphis morvus morvus (Fabricius, 1775)

Memphis philumena corita (Fruhstorfer, 1916)

Mesoprepona pheridamas (Cramer, 1777)

Prepona laertes (Hübner, [1811])

Prepona laertes octavia Fruhstorfer, 1905

Prepona omphale (Hübner, 1819)

Prepona pheridamas (Cramer, 1777)

Prepona pseudomphale Le Moult, 1932

Siderone galanthis (Cramer, 1755)

Zaretis isidora (Cramer, 1779)

$\mathrm{X}$

Zaretis itys (Cramer, 1777)

\section{Cyrestinae}

Marpesia chiron (Fabricius, 1775)

Marpesia petreus (Cramer, 1776)

\section{Danainae}

Danaus eresimus (Cramer, 1777)

Danaus gilippus (Cramer, 1775)

Danaus vanillae vanillae (Linnaeus, 1758)

Danaus sp.

Lycorea halia (Hübner, 1816)

Methona sp.

Heliconiinae

Agraulis vanillae (Linnaeus, 1758)

Dione vanillae vanillae (Linnaeus, 1758)

$\mathrm{x}$

Dione sp.

Dryadula phaetusa (Linnaeus, 1758)

Dryas iulia (Fabricius, 1775)

Dryas sp.

Eueides lybia (Fabricius, 1775)

Euptoieta hegesia (Cramer, 1779)

Euptoieta sp.

Heliconius antiochus (Linnaeus, 1767)

Heliconius doris (Linnaeus 1771)

Heliconius erato phyllis (Fabricius, 1775)

Heliconius melpomene (Linnaeus, 1758)

$\mathrm{x}$

$\mathrm{x}$ 
Tabela 3. Continuação...

\begin{tabular}{|c|c|c|c|c|c|c|}
\hline Espécies & Bates (1867) & $\begin{array}{l}\text { Garcia et } \\
\text { al.(1990) }\end{array}$ & $\begin{array}{c}\text { Garcia \& } \\
\text { Bergmann } \\
\text { (1994) }\end{array}$ & $\begin{array}{l}\text { Ramos } \\
(2000)\end{array}$ & $\begin{array}{l}\text { Martins et } \\
\text { al. (2017) }\end{array}$ & $\begin{array}{l}\text { Presente } \\
\text { estudo }\end{array}$ \\
\hline Heliconius melpomene melpomene (Linnaeus, 1758) & & & & & $\mathrm{x}$ & \\
\hline Heliconius melpomene nanna Stichel, 1899 & & & & & $\mathrm{x}$ & \\
\hline Heliconius novatus Bates, 1862 & $\mathrm{x}$ & & & & & \\
\hline Heliconius numata (Cramer, 1780) & & & & & $\mathrm{x}$ & $\mathrm{x}$ \\
\hline Heliconius paraplesius $\mathrm{H}$. Bates, 1867 & & & & & & $\mathrm{x}$ \\
\hline Heliconius ricini (Linnaeus, 1758) & & & & & $\mathrm{x}$ & \\
\hline Heliconius sara sara (Fabricius, 1793) & & & & & $\mathrm{x}$ & $\mathrm{x}$ \\
\hline Heliconius sp. 1 & & $\mathrm{x}$ & & & & \\
\hline
\end{tabular}

Heliconius sp. 2

Heliconius sp. 3

$\mathrm{X}$

Heliconius wallacei flavescens Weymer, 1891

Leptalis orise Bates, 1867

$\mathrm{x}$

Leptalis Theonoe Bates, 1867

Neruda mentis Moreira \& Mielke, 2010

Philaethria dido (Linnaeus, 1763)

Philaethria sp.

$\mathrm{x}$

Ithomiinae

Ceratinia sp.

Episcada sp.

Hypothyris euclea (Godart, 1819)

Hypothyris sp.

Mechanitis lysimnia (Fabricius, 1793)

Mechanitis polymnia (Linnaeus, 1758)

Methona sp.

Napeogenes sp.

Oleria sp.

Scada sp.

Limenitidinae

Adelpha cytherea cytherea (Linnaeus, 1758)

Adelpha iphiclus iphiclus (Linnaeus, 1758)

Adelpha sp. 1

Adelpha sp. 2

Morphinae

Morpho achilles (Linnaeus, 1758)

Morpho deidamia Hübner, 1819

Morpho helenor (Cramer, 1776)

Morpho menelaus terrestris (Butler, 1866)

Morpho rhetenor Butler, 1866

x

$x$

Nymphalinae

Anartia amathea (Linnaeus, 1758)

Anartia jatrophae (Linnaeus, 1763)

Colobura dirce (Linnaeus, 1758)

Colobura sp.

Historis acheronta (Fabricius, 1775)

Historis odius (Fabricius, 1775)

Junonia evarete (Cramer, 1779)

Junonia sp.

Junonia sp.1

$\mathrm{X}$

$\mathrm{x}$

$\mathrm{x}$

\begin{tabular}{|c|c|c|c|c|c|}
\hline 8) & & & $\mathrm{x}$ & & \\
\hline 9 & & & & & $\mathrm{x}$ \\
\hline & $\mathrm{x}$ & $\mathrm{x}$ & $\mathrm{x}$ & $\mathrm{x}$ & $\mathrm{x}$ \\
\hline atler, 1866) & & & & $x$ & $\mathrm{x}$ \\
\hline
\end{tabular}


Tabela 3. Continuação...

\begin{tabular}{|c|c|c|c|c|c|c|}
\hline Espécies & Bates (1867) & $\begin{array}{l}\text { Garcia et } \\
\text { al.(1990) }\end{array}$ & $\begin{array}{c}\text { Garcia \& } \\
\text { Bergmann } \\
\text { (1994) }\end{array}$ & $\begin{array}{l}\text { Ramos } \\
\text { (200o) }\end{array}$ & $\begin{array}{l}\text { Martins et } \\
\text { al. (2017) }\end{array}$ & $\begin{array}{c}\text { Presente } \\
\text { estudo }\end{array}$ \\
\hline
\end{tabular}

Siproeta stelenes (Linnaeus, 1758)

$\mathrm{x}$

Tigridia acesta (Linnaeus, 1758)

$\mathrm{x}$ $\mathrm{x}$

\section{Satyrinae}

Caeruleuptychia brixius (Godart, [1824])

$\mathrm{x}$

Cepheuptychia cephus (Fabricius, 1755)

Cepheuptychia coelestes (Butler, 1860)

Chloreuptychia arnaca (Fabricius, 1777)

Chloreuptychia chloris (Cramer, 1782)

Chloreuptychia herseis (Godart, 1824])

Caeruleuptychia penicillata (Godman, 1905)

Caeruleuptychia sp.

Cissia "bege"

Cissia myncea (Cramer, 1780)

Cissia palladia (Butler, 1867)

Cissia occypede (Fabricius, 1777)

Cissia penelope (Fabricius, 1775)

Cissia terrestris (Butler, 1866)

Erichthodes erichtho (Butler, 1866)

Erichthodes numeria (Felder, 1865)

Harjesia blanda (Möschler, 1876)

Harjesia sp.

Hermeuptychia hermes (Fabricius, 1775)

Hermeuptychia sp.

Magneuptychia alcinoe (C. \& R. Felder, 1867)

Magneuptychia libye (Linnaeus, 1767)

Magneuptychia ocypete (Fabricius, 1776)

Magneuptychia pallema (Schaus, 1902)

Magneuptychia sp..

Pareuptychia metaleuca (Boisduval, 1870)

Pareuptychia ocirrhoe (Fabricius, 1776)

Paryphthimoides phronius (Godart, [1824])

Paryphthimoides undulata (Butler, 1867)

Pharneuptychia innocentia (C. Felder \& R. Felder, 1867)

Pharneuptychia phares (Godart, [1824])

Pharneuptychia pharnaces (Weymer, 1911)

Pierella hyalinus (Gmelin, [1790])

Pierella lamia (Sulzer, 1776)

Posttaygetis penelea (Cramer, 1777)

Pseudodebis valentina (Cramer, 1779)

Selenophanes cassiope (Cramer, 1775)

Taeygetis sosis Hopffer, 1874

Taygetina kerea (A. Butler, 1869)

Taygetis celia (Cramer, 1782)

Taygetis cleopatra C. Felder \& R. Felder, 1867

Taygetis echo (Cramer, 1775)

Taygetis laches Fabricius, 1793

Taygetis larua C. Felder \& R. Felder, 1867

Taygetis leuctra A. Butler, 1870

Taygetis mermeria (Cramer, 1776)

Taygetis virgilia (Cramer, 1776) $\mathrm{x}$

$\mathrm{x}$

$\mathrm{x}$

$\mathrm{X}$

$\mathrm{x}$

$\mathrm{x}$

$\mathrm{X}$

$\mathrm{X}$

$\mathrm{X}$

$\mathrm{x}$

$\mathrm{x}$

$\mathrm{x}$

$\mathrm{x}$

$\mathrm{x}$

$\mathrm{X}$

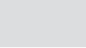

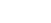

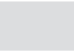

$x$
$x$

$\mathrm{x}$

$\mathrm{x}$

$\mathrm{x}$

$\mathrm{x}$

$\mathrm{x}$

$\mathrm{x}$

$\mathrm{x}$

$\mathrm{x}$

$\mathrm{x}$

$\mathrm{x}$

Continua.. 
Tabela 3. Continuação...

\begin{tabular}{|c|c|c|c|c|c|c|}
\hline Espécies & Bates (1867) & $\begin{array}{l}\text { Garcia et } \\
\text { al.(1990) }\end{array}$ & $\begin{array}{c}\text { Garcia \& } \\
\text { Bergmann } \\
(1994)\end{array}$ & $\begin{array}{l}\text { Ramos } \\
(2000)\end{array}$ & $\begin{array}{l}\text { Martins et } \\
\text { al. }(2017)\end{array}$ & $\begin{array}{c}\text { Presente } \\
\text { estudo }\end{array}$ \\
\hline Taygetis thamyra (Cramer, 1779) & & & & & & $\mathrm{x}$ \\
\hline Taygetis virgilia (Cramer, 1776) & & & & $\mathrm{x}$ & & $x$ \\
\hline Yphthimoides affinis (Butler, 1867) & & & & & $\mathrm{x}$ & \\
\hline Yphthimoides renata (Stoll, 1780) & & & & & $\mathrm{x}$ & \\
\hline Yphthimoides sp. & & & & $\mathrm{x}$ & & \\
\hline Yphthimoides disaffecta (Butler \& Druce, 1874) & & & & $\mathrm{x}$ & & \\
\hline
\end{tabular}

\section{Polyommatinae}

\section{Coliadinae}

Anteos menippe (Hübner, [1818])

Anteos sp. 1

$\mathrm{X}$

Anteos sp. 2

Aphrissa statira statira (Cramer, 1777)

Eurema albula (Cramer, 1775)

Eurema deva (Doubleday \& Hewitson, 1850)

Eurema elathea (Cramer, 1777)

Eurema sp.

Phoebis argante argante (Fabricius, 1775)

Phoebis philea philea (Linnaeus, 1763)

Phoebis sennae marcellina (Cramer, 1777)

Phoebis sennae sennae (Linnaeus, 1758)

Phoebis sp.

Pyrisitia nise (Cramer, 1775)

Polyommatinae

Arawacus sp.

Evenus sp.

Hemiargus hanno hanno (Stoll, 1790)

Hemiargus sp.

$\mathrm{x}-\mathrm{x}$

$\mathrm{x}$

$\mathrm{x}$

$\mathrm{x}$

$\mathrm{x}-\mathrm{x}$

$\mathrm{x}$

Leptotes cassius (Cramer, 1775)

\section{Theclinae}

Arawacus aetolus (Sulzer, 1776)

Calycopis demonassa (Hewitson, 1868)

Chlorostrymon telea (Hewitson, 1868)

Evenus satyroides (Hewitson,1865)

Iaspis castitas (H. H. Druce, 1907)

Ministrymon megacles (Stoll, 1780)

Ministrymon zilda (Hewitson, 1873)

Nicolaea socia (Hewitson, 1868)

Panthiades phaleros (Linnaeus, 1767)

Pseudolycaena marsyas (Linnaeus, 1758)

Rekoa palegon (Cramer, 1780)

Strymon mulucha (Hewitson, 1867)

Tmolus echion (Linnaeus, 1767)

\section{Pieridae}

\section{Coliadinae}

Anteos menippe (Hübner, [1818])

Aphrissa statira (Cramer, 1777)

Eurema agave (Cramer, 1775)

Eurema albula (Cramer, 1775)

Eurema elathea (Cramer, 1777)

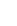

$\sqrt{2}+x^{2}$

$x^{2}$


Tabela 3. Continuação...

\begin{tabular}{|c|c|c|c|c|c|c|}
\hline Espécies & Bates (1867) & $\begin{array}{l}\text { Garcia et } \\
\text { al.(1990) }\end{array}$ & $\begin{array}{c}\text { Garcia \& } \\
\text { Bergmann } \\
(1994)\end{array}$ & $\begin{array}{l}\text { Ramos } \\
(2000)\end{array}$ & $\begin{array}{c}\text { Martins et } \\
\text { al. (2017) }\end{array}$ & $\begin{array}{c}\text { Presente } \\
\text { estudo }\end{array}$ \\
\hline Phoebis argante (Fabricius, 1775) & & & & & & $\mathrm{x}$ \\
\hline
\end{tabular}

\section{Pierinae}

Ascia monuste (Linnaeus, 1764)

$\mathrm{x}$

Glutophrissa drusilla (Cramer, 1777)

Itaballia demophile (Linnaeus, 1763)

\section{RIODINIDAE}

\section{Riodininae}

Amarynthis meneria (Cramer, 1776

Apodemia sp.

Aricoris campestris (Bates, 1868)

Aricoris propitia (Stichel, 1910)

Baeotis euprepes (Bates, 1868)

Calospila lucianus (Fabricius, 1793)

Calospila sp.

Calydna sp.

Charis sp.

Detritivora zama (Bates, 1868)

Emesis diogenia Prittwitz, 1865

Esthemopsis sericina (Bates, 1867)

Eurybia elvina Stichel,1910

Eurybia patrona Weymer, 1875

Helicopis cupido (Linnaeus, 1758)

Isapis agyrtus (Cramer, 1777)

Juditha odites odites (Cramer, 1775)

Lemonias zygia Hübner, 1807

Melanis smithiae (Westwood, 1851)

Mesene phareus (Cramer, 1777)

Mesosemia sp.

Mesosemia steli Hewitson 1858

Napaea actoris Bates, 1862

Napaea beltiana beltiana (Bates, 1867)

Napaea eucharila (Bates, 1867)

Napaea heteroea Bates, 1867

Napaea melampia (Bates, 1867)

Napaea phryxe (C. \& R. Felder, 1865)

Nymphidium chione (Bates, 1867)

Nymphidium sp.

Nymphidium sylvarum Bates, 1862

Phaenochitonia cingulus (Stoll, 1790)

Setabis sp.

Stalachtis phlegia (Cramer, 1779)

Stalachtis sp. 1

Stalachtis sp. 2

Stalachtis sp. 3

Synargis agle (Hewitson, [1853])

Synargis axenus axenus (Hewitson, 1876)

Synargis calyce (C. Felder \& R. Felder, 1862)

Synargis galena (Bates, 1868)

Synargis gela (Hewitson, [1853]) $\mathrm{x}$

$\mathrm{x}$

$\mathrm{x}$

$\mathrm{x}$

$\mathrm{x}$

$\mathrm{x}$

$\mathrm{x}$

$x$

$\mathrm{x}$

$\mathrm{x}$

$\mathrm{x}$

$\mathrm{x}$

$\mathrm{x}$

$\mathrm{x}$

$\mathrm{x}$

$\mathrm{x}$

$\mathrm{x}$

$\mathrm{x}$

$\mathrm{x}$

$\mathrm{x}$

$\mathrm{x}$

$\mathrm{x}$

$\mathrm{x}$

$\mathrm{x}$

$\mathrm{x}$

$\mathrm{x}$

Continua... 
Tabela 3. Continuação...

\begin{tabular}{|c|c|c|c|c|c|c|}
\hline Espécies & Bates (1867) & $\begin{array}{l}\text { Garcia et } \\
\text { al.(1990) }\end{array}$ & $\begin{array}{c}\text { Garcia \& } \\
\text { Bergmann } \\
\text { (1994) }\end{array}$ & $\begin{array}{l}\text { Ramos } \\
\text { (2000) }\end{array}$ & $\begin{array}{l}\text { Martins et } \\
\text { al. (2017) }\end{array}$ & $\begin{array}{c}\text { Presente } \\
\text { estudo }\end{array}$ \\
\hline Theope foliorum Bates, 1868 & & & & & $\mathrm{x}$ & \\
\hline Theope pieridoides C. Felder \& R. Felder, 1865 & & & & & & $\mathrm{x}$ \\
\hline Theope sp. & & & & & & $\mathrm{x}$ \\
\hline Thisbe irenea (Stoll, 1780) & & & & & $\mathrm{x}$ & \\
\hline Thisbe molela (Hewitson, 1865) & & & & & $\mathrm{x}$ & \\
\hline
\end{tabular}

\section{Suggestion citation:}

Pereira, S., W.M.A. Rezende \& J.T. Câmara, 2018. Borboletas (Lepidoptera: Papilionoidea e Hesperioidea) da Reserva Biológica do Gurupi, Maranhão, Brasil. EntomoBrasilis, 11 (2): 124-138.

Available on: doi:10.12741/ebrasilis.v11i2.759
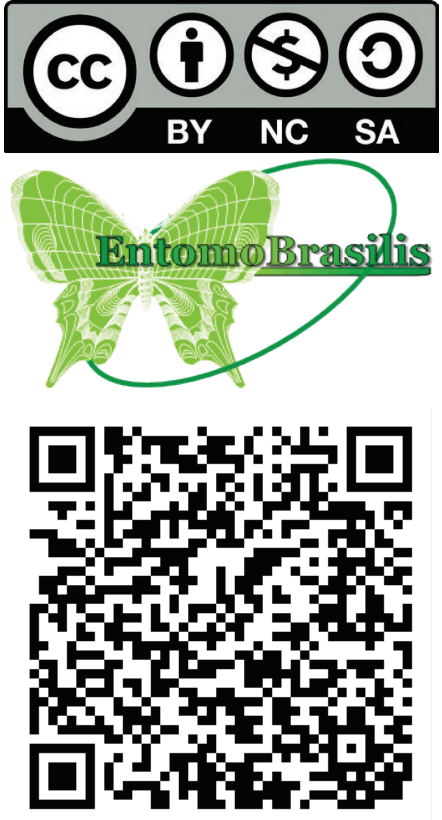